\title{
Short-term outcomes of a simple and effective approach to aortic root and arch repair in acute type $A$ aortic dissection
}

\author{
Bo Yang, MD, PhD, ${ }^{\text {a }}$ Aroosa Malik, BS, ${ }^{\text {a }}$ Victoria Waidley, BS, ${ }^{\mathrm{a}}$ Kellianne C. Kleeman, MD, \\ Xiaoting Wu, PhD, ${ }^{a}$ Elizabeth L. Norton, MS, ${ }^{\mathrm{b}}$ David M. Williams, MD, \\ Minhaj S. Khaja, MD, MBA, ${ }^{c}$ and Whitney E. Hornsby, $\mathrm{PhD}^{\mathrm{b}}$
}

\section{ABSTRACT}

Objective: To evaluate short-term outcomes following direct aortic root and arch repair in patients with acute type A aortic dissection (ATAAD) without technical adjuncts.

\begin{abstract}
Methods: Between 2012 and 2016, 94 consecutive patients with ATAAD underwent surgical repair, including aortic root repair $(n=45)$, root replacement $(\mathrm{n}=39)$, or no root procedure $(\mathrm{n}=10)$. Aortic root repair was achieved by running approximation of the dissected aortic wall circumferentially at the sinotubular junction and reinforcing the coronary ostia with 5-0 Prolene. The aortic root and arch were anastomosed to the Dacron graft with 5-0 Prolene without Teflon felt or biological glue.
\end{abstract}

Results: Postoperative new-onset myocardial infarction, stroke, renal failure, and complete heart block occurred in $0 \%, 4 \%, 13 \%$, and $0 \%$ of patients, respectively, whereas 30-day mortality was $4 \%$. The incidences of permanent neurologic deficit and renal failure were $1 \%$ and $2 \%$, respectively. Up to 5 years, the aortic root repair group was free from residual or recurrent aortic root dissection, major change in the aortic root diameter, and moderate to severe aortic regurgitation; the entire cohort was free of anastomotic pseudoaneurysm and reoperation for proximal aortic pathology or significant change in diameter of the aortic arch and descending thoracic aorta. Overall survival was $85 \%$ at 4 years and was significantly enhanced in the aortic root repair group compared with the Bentall group $(\mathrm{n}=24)(93 \%$ vs $57 \% ; P=.035)$.

Conclusions: Direct aortic root and arch repair with approximation of the aortic wall without use of technical adjuncts is safe and effective for patients with ATAAD. If warranted, preservation of the native aortic valve should be considered for a potential survival benefit. (J Thorac Cardiovasc Surg 2018;155:1360-70)

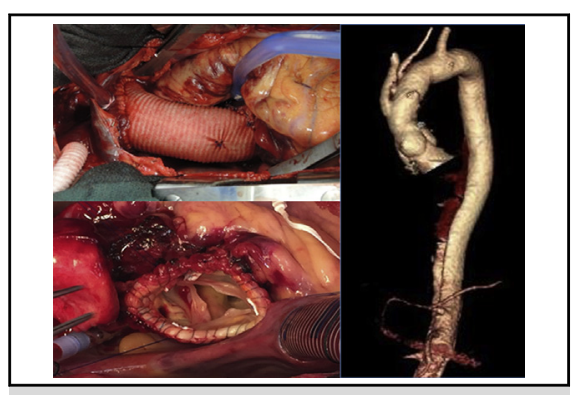

Direct repair of a dissected aortic root and arch without the use of technical adjuncts.

\section{Central Message}

Direct repair of the aortic root and arch without use of Teflon felt or biological glue is safe and effective in patients with acute type A aortic dissection.

\section{Perspective}

For patients with acute type A aortic dissection, direct aortic root repair with coronary ostia reinforcement and arch repair without the use of Teflon felt or biological glue is safe and effective. There was low 30-day mortality (4\%) and no recurrent proximal aortic pathology or reoperation up to 5 years. Short-term survival was enhanced in the aortic root repair group compared with the Bentall group.

See Editorial Commentary page 1371.

See Editorial page 1355.
Acute type A aortic dissection (ATAAD) is a devastating condition carrying a high risk of operative morbidity and mortality. At present, 30-day mortality remains between $20 \%$ and $25 \%$. Surgical repair of an ATAAD is a technical

From the Departments of a Cardiac Surgery, ${ }^{\mathrm{b}}$ Internal Medicine, and ${ }^{\mathrm{c}}$ Radiology, Michigan Medicine, Ann Arbor, Mich.

This study was supported by the Phil Jenkins Breakthrough Fund, Steven J. Szatmari and Darlene Szatmari, and Michigan Medicine, Department of Cardiac Surgery.

Received for publication March 20, 2017; revisions received Nov 16, 2017; accepted for publication Nov 28, 2017; available ahead of print Feb 2, 2018.

Address for reprints: Bo Yang, MD, PhD, 1500 East Medical Center Dr, 5155 Frankel Cardiovascular Center, Ann Arbor, MI 48109 (E-mail: boya@med.umich.edu). $0022-5223 / \$ 36.00$

Copyright (c) 2017 by The American Association for Thoracic Surgery

https://doi.org/10.1016/j.jtcvs.2017.11.089 challenge due to the fragility of the aorta as well as the inflammation and hematoma surrounding the aorta. Over the past 20 years, biological glue and Teflon felt have been used extensively to facilitate ATAAD repair and anastomosis of the aorta.

Despite the acceptance of biological glue and Teflon felt for ATAAD repair, early and late complications

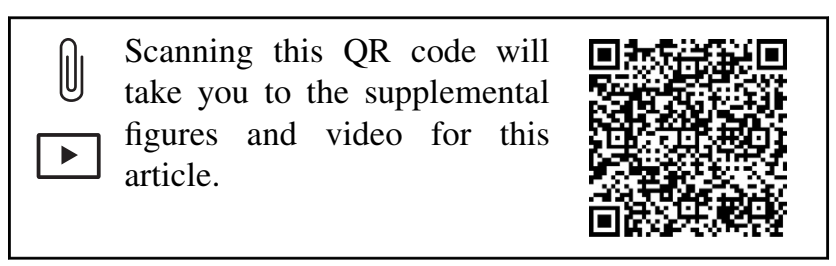



Abbreviations and Acronyms
ATAAD $=$ acute type A aortic dissection
$\mathrm{CI}=$ confidence interval
$\mathrm{CTA}=$ computed tomography angiography
HCA = hypothermia circulatory arrest
HIPAA $=$ Health Insurance Portability and
Accountability Act
MI = myocardial infarction
PRBCs = packed red blood cells
STJ = sinotubular junction

\section{Patient Selection}

Between 2012 and 2016, 94 consecutive patients underwent direct aortic root or arch repair with 5-0 Prolene suture and no adjuncts (ie, Teflon felt or biological glue) as a new method for surgical treatment of ATAAD. The patients were divided into 3 groups based on the aortic root procedure: aortic root repair $(\mathrm{n}=45)$, aortic root replacement $(\mathrm{n}=39)$ performed with either the David procedure $(n=15)$ or Bentall procedure $(n=24)$, and no root intervention $(\mathrm{n}=10)$ owing to the absence of aortic root pathology $(n=9)$ or previous aortic root replacement $(n=1)$. Society of Thoracic Surgery (STS) data were obtained from Michigan Medicine's Department of Cardiac Surgery data warehouse to identify the cohort and to determine preoperative, operative, and postoperative characteristics. Medical record review was performed to supplement data collection. All of the operative reports were reviewed by one of the surgical investigators (B.Y.) to confirm the diagnoses and the procedures.

All patients were followed after aortic root repair with CTA of the aorta and/or echocardiography at 3 months, 1 year, and then every 1 to 2 years depending on the presence of residual aortic dissection and progression of the aortic aneurysm at our center. For patients receiving outside follow-up care, the same strategy was implemented, and medical records were received by our center. All living patients were mailed an Institutional Review Board-approved questionnaire or contacted by phone to obtain current health information regarding subsequent reoperations on the aorta. The National Death Index database was used to supplement data on survival through $2015 .^{10}$

\section{Operative Technique (Video 1 )}

Our criteria for direct aortic root repair included no intimal tear at the root, no connective tissue disease, and root diameter $<4 \mathrm{~cm}$ in patients age $\leq 65$ years and $<4.5 \mathrm{~cm}$ in those age $>65$ years. ${ }^{8,11,12}$ Patients meeting the criteria for root repair without aortic valve pathology were managed with aortic root repair. Patients meeting the criteria for aortic root repair with aortic valve pathology were managed with aortic root and aortic valve repair or total aortic root replacement based on whether

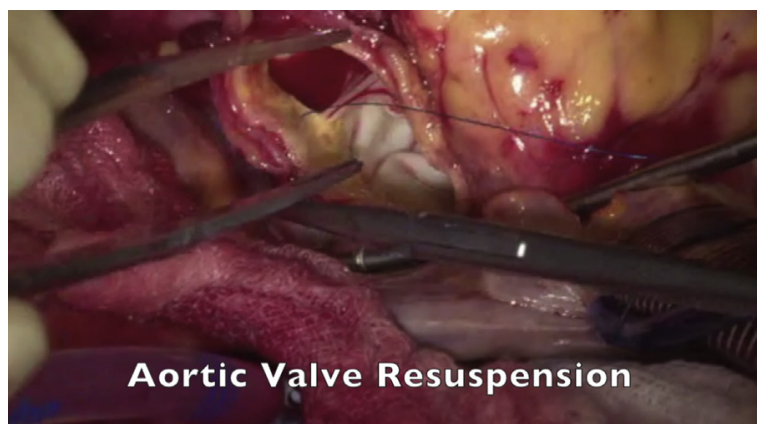

VIDEO 1. The technique of direct repair of the aortic root and arch with fine sutures and no surgical technical adjuvants, such as biological glue or Teflon filter, in an 82-year-old female with acute type A aortic dissection. The aortic dissection was complicated with right coronary artery dissection, intraoperative propagation of aortic dissection to the branches of the aortic arch and distal aorta, and new-onset malperfusion of the lower body during the operation. Direct aortic root repair, transverse arch replacement, and right coronary bypass were performed. After aortic root repair, a transesophageal echocardiogram showed obliteration of the false lumen of the dissected aortic root and a competent aortic valve with no aortic insufficiency. The patient was discharged on postoperative day 8 without any complications. Video available at: http://www.jtcvsonline.org/article/ S0022-5223(17)32851-9/fulltext. 

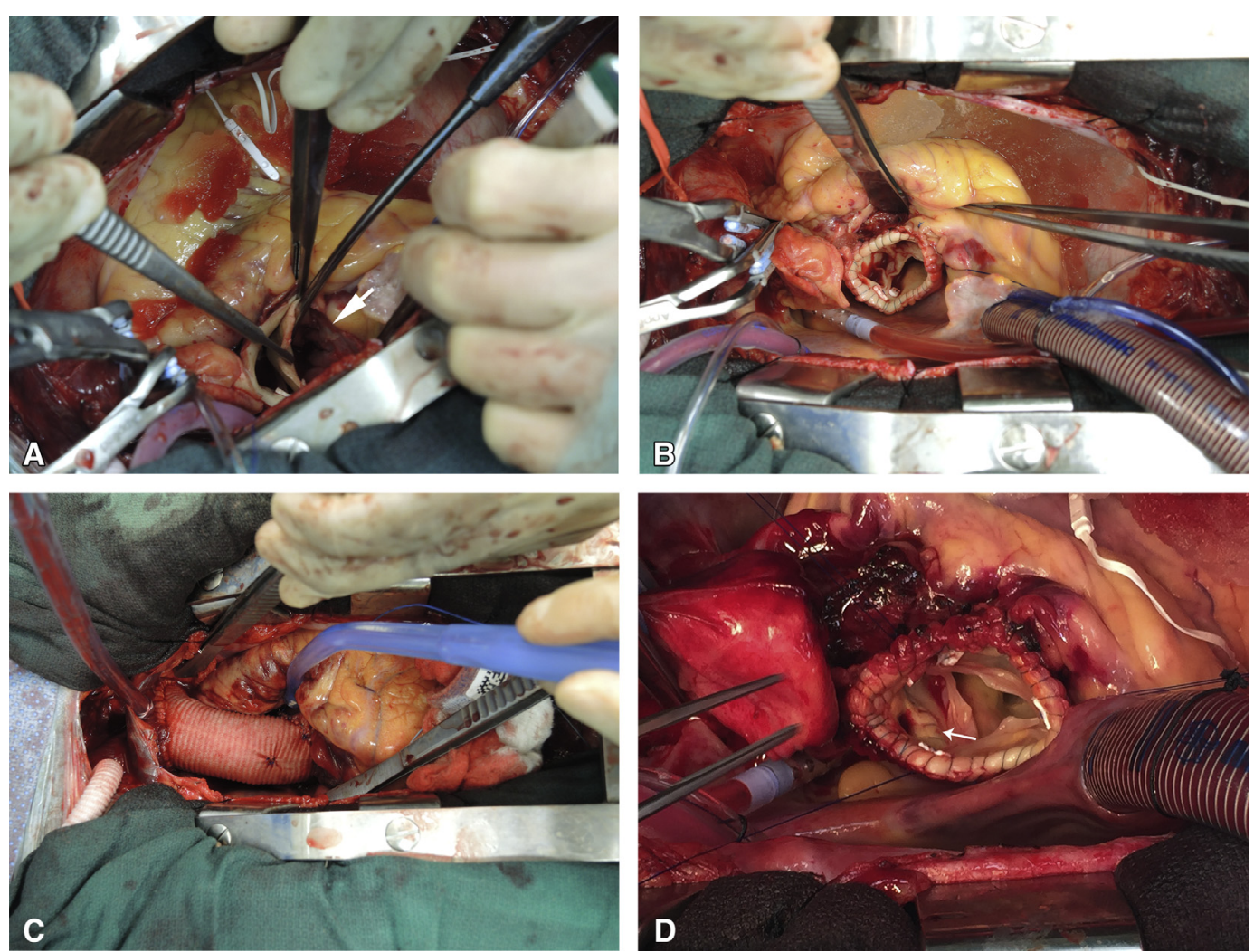

FIGURE 1. Direct repair of aortic root and arch in acute type A aortic dissection. A, Dissected noncoronary sinus of the aortic root (arrow, false lumen). B, Root repair with 5-0 Prolene circumferential running suture. C, Completion of repair with an interpositional Dacron graft telescope into the true lumen of the dissected aorta at the sinotubular junction and the arch with 5-0 Prolene running suture. D, The left coronary sinus wall was reinforced around the coronary ostium with 5-0 Prolene as an in situ coronary button reimplantation in a different case with aortic dissection around the coronary ostia.

valve repair was technically feasible. The criteria of direct repair of a dissected aortic arch with transverse arch replacement but without replacement of arch branch vessels included no intimal tear at the arch, arch diameter $<4 \mathrm{~cm}$, and no malperfusion of innominate artery or left common carotid artery. In younger patients, if the innominate artery or left common carotid artery was dissected, we replaced the arch and the innominate artery or left common carotid artery with a multibranch Dacron graft (Hemashield; Marquet, Boston, Mass). If the entire common carotid artery was dissected and partially or completely occluded, we replaced the entire dissected common carotid arteries with separate neck incisions to the common carotid bifurcation.

To directly repair the aortic root, we trimmed the aortic root 3 to $5 \mathrm{~mm}$ above the sinotubular junction (STJ) or coronary ostia, whichever was more distal, and removed all of the thrombus in the false lumen at each aortic sinus (Figure 1, A). We reapproximated the aortic wall at the STJ with a 5-0 Prolene in running suture fashion circumferentially (Figure $1, B$ ). Next, the aortic valve was resuspended at the 3 commissural posts with pledgeted 4-0 Prolene suture. If the left or right coronary sinus wall was dissected around the coronary ostia, the aortic sinus wall was reinforced around the coronary ostia with an in situ full-thickness running 5-0 Prolene suture in the same fashion as coronary button reimplantation in the Bentall or David procedure (Figure 1, D). However, if the dissection extended into the coronary arteries, coronary artery bypass was performed instead. If the small intimal tear from the STJ extended into the coronary ostium (most often the left coronary ostium), the intimal tear was repaired with 5-0 Prolene, with full-thickness bites in either interrupted or running suture fashion before closing the false lumen at the STJ. The repaired aortic root was then anastomosed to the Dacron graft with a running 5-0 Prolene suture as the proximal anastomosis (Figure 1, C). The distal anastomosis at the aortic arch with the Dacron graft was also completed with a running 5-0 Prolene suture without biological glue or Teflon felt after all of the thrombus was removed from the false lumen at the anastomotic site (Figure 1,C). It is important that the Dacron graft is telescoped into the aortic true lumen at both proximal and distal anastomoses and to keep the Dacron graft in contact with the intima of the aorta. As long as the criteria of aortic root repair were met, all aortic sinuses of the aortic root were preserved, to maintain the normal geometry of the aortic root even if the aortic dissection extended down to the aortic annulus. All arch replacements were performed with moderate hypothermia circulatory arrest, with bladder temperature maintained at $24^{\circ} \mathrm{C}$ to $28^{\circ} \mathrm{C}$ supplemented with unilateral antegrade cerebral perfusion. No Teflon felt, biological glue, or any other adjuncts were used for any anastomosis or repair.

\section{Statistical Analysis}

Data are presented as median (interquartile range) for continuous data and as number $(\%)$ for categorical data. Univariate comparisons across different groups of aortic root procedures (aortic root repair vs root replacement) were performed using the $\chi^{2}$ test for categorical data and the Wilcoxon rank-sum test for continuous data. Stepwise logistic regression was used to report adjusted odds ratios (ORs) with $95 \%$ confidence intervals (CIs) for operative mortality. Crude survival curves were estimated using the nonparametric Kaplan-Meier method. The log-rank test was used to compare survival between groups (native aortic valve vs prosthetic aortic valve). Cox proportional hazards regression 
TABLE 1. Demographic and preoperative data

\begin{tabular}{|c|c|c|c|c|c|}
\hline Variables & Total $(n=94)$ & Root repair $(n=45)$ & Root replacement $(\mathbf{n}=39)$ & $\begin{array}{c}\text { No root procedure } \\
(\mathbf{n}=\mathbf{1 0})\end{array}$ & $P$ value \\
\hline Patient age, y, median (IQR) & $59(52-66)$ & $60(54-66)$ & $57(46-65)$ & $60(51-71)$ & .216 \\
\hline Female sex, n (\%) & $29(31)$ & $20(44)$ & $5(13)$ & $4(40)$ & .002 \\
\hline \multicolumn{6}{|l|}{ Preexisting comorbidities, n (\%) } \\
\hline Diabetes mellitus & $8(9)$ & $3(7)$ & $5(13)$ & $0(0)$ & .463 \\
\hline Hypertension & $67(71)$ & $34(76)$ & $26(67)$ & $7(70)$ & .368 \\
\hline Coronary artery disease & $8(9)$ & $1(2)$ & $7(18)$ & $0(0)$ & .022 \\
\hline Smoking & & & & & .910 \\
\hline Never & $49(52)$ & $22(49)$ & $20(51)$ & $7(70)$ & \\
\hline Current & $25(27)$ & $13(29)$ & $9(23)$ & $3(30)$ & \\
\hline Former & $16(17)$ & $8(18)$ & $8(21)$ & $0(0)$ & \\
\hline Missing & $4(4)$ & $2(4)$ & $2(5)$ & $0(0)$ & \\
\hline COPD & $15(16)$ & $7(16)$ & $7(18)$ & $1(10)$ & .769 \\
\hline Stroke & $10(11)$ & $6(13)$ & $3(8)$ & $1(10)$ & .494 \\
\hline TIA & $3(3)$ & $2(4)$ & $1(3)$ & $0(0)$ & 1.000 \\
\hline MFS & $3(3)$ & $0(0)$ & $2(5)$ & $1(10)$ & .213 \\
\hline Peripheral vascular disease & $5(5)$ & $1(2)$ & $3(8)$ & $1(10)$ & .333 \\
\hline Aortic valve morphology, n (\%) & & & & & 081 \\
\hline Trileaflet & $86(91)$ & $44(98)$ & $33(85)$ & $9(90)$ & \\
\hline Bicuspid and unicuspid & $5(5)$ & $1(2)$ & $4(10)$ & $0(0)$ & \\
\hline Prosthesis & $3(3)$ & $0(0)$ & $2(5)$ & $1(10)$ & \\
\hline Previous cardiac surgery, n (\%) & $10(11)$ & $1(2)$ & $8(21)$ & $1(10)$ & .01 \\
\hline Preoperative AI, n (\%) & & & & & .001 \\
\hline None & $25(27)$ & $8(18)$ & $12(31)$ & $5(50)$ & \\
\hline Trace & $10(11)$ & $8(18)$ & $1(3)$ & $1(10)$ & \\
\hline Mild & $30(32)$ & $19(42)$ & $7(18)$ & $4(40)$ & \\
\hline Moderate & $16(17)$ & $7(16)$ & $9(23)$ & $0(0)$ & \\
\hline Severe & $13(14)$ & $3(7)$ & $10(26)$ & $0(0)$ & \\
\hline Acute myocardial infarction, $\mathrm{n}(\%)$ & $3(3)$ & $1(2)$ & $2(5)$ & $0(0)$ & .595 \\
\hline History of chronic renal disease, $\mathrm{n}(\%)$ & $6(6)$ & $2(4)$ & $4(10)$ & $0(0)$ & 1.000 \\
\hline History of renal failure on dialysis, $\mathrm{n}(\%)$ & $3(3)$ & $1(2)$ & $2(5)$ & $0(0)$ & .599 \\
\hline Acute kidney injury, n (\%) & $14(15)$ & $4(9)$ & $8(21)$ & $2(20)$ & .129 \\
\hline Preoperative creatinine, $\mathrm{mg} / \mathrm{dL}$, median (IQR) & $1.1(0.9-1.3)$ & $1.1(0.9-1.3)$ & $1.2(0.9-1.4)$ & $1.1(0.9-1.2)$ & .637 \\
\hline NYHA functional class, n (\%) & & & & & .909 \\
\hline I & $84(89)$ & $41(91)$ & $35(90)$ & $8(80)$ & \\
\hline II & $5(5)$ & $3(7)$ & $2(5)$ & $0(0)$ & \\
\hline III & $2(2)$ & $0(0)$ & $1(3)$ & $1(10)$ & \\
\hline IV & $3(3)$ & $1(2)$ & $1(3)$ & $1(10)$ & \\
\hline Ejection fraction, $\%$, median (IQR) & $55(55-60)$ & $60(55-62.5)$ & $55(55-60)$ & $60(52.5-70)$ & .267 \\
\hline Malperfusion, n (\%) & $30(32)$ & $17(38)$ & $12(31)$ & $1(10)$ & .50 \\
\hline Cerebral & $5(5)$ & $3(7)$ & $2(5)$ & $0(0)$ & .68 \\
\hline Coronary & $2(2)$ & $0(0)$ & $2(5)$ & $0(0)$ & 213 \\
\hline Mesentery & $8(9)$ & $4(9)$ & $3(8)$ & $1(10)$ & 1.000 \\
\hline Renal & $10(11)$ & $6(13)$ & $3(8)$ & $1(10)$ & 494 \\
\hline Extremities & $23(24)$ & $13(29)$ & $9(23)$ & $1(10)$ & .546 \\
\hline Spinal cord & $2(2)$ & $2(4)$ & $0(0)$ & $0(0)$ & .497 \\
\hline Preoperative fenestration and stenting, $\mathrm{n}(\%)$ & $8(9)$ & $3(7)$ & $4(10)$ & $1(10)$ & .699 \\
\hline Aortic rupture, n (\%) & $10(11)$ & $4(9)$ & $5(13)$ & $1(10)$ & .727 \\
\hline Cardiac tamponade, n (\%) & $15(16)$ & $11(24)$ & $1(3)$ & $3(30)$ & 004 \\
\hline Shock, n (\%) & $15(16)$ & $7(16)$ & $5(13)$ & $3(30)$ & .721 \\
\hline
\end{tabular}


TABLE 1. Continued

\begin{tabular}{lccccc}
\hline \multicolumn{1}{c}{ Variables } & Total $(\mathbf{n}=\mathbf{9 4})$ & Root repair $(\mathbf{n}=\mathbf{4 5})$ & Root replacement $(\mathbf{n}=\mathbf{3 9 )}$ & \multicolumn{2}{c}{ No root procedure } \\
$(\mathbf{n}=\mathbf{1 0})$ & $\boldsymbol{P}$ value \\
\hline Anticoagulant use, $\mathrm{n}(\%)$ & $11(12)$ & $1(2)$ & $8(21)$ & $2(20)$ & .011 \\
Preoperative inotropic support, $\mathrm{n}(\%)$ & $8(9)$ & $4(9)$ & $2(5)$ & .681 \\
Preoperative cardiac arrest, $\mathrm{n}(\%)$ & $3(3)$ & $1(2)$ & $2(5)$ & $.20)$ \\
\hline
\end{tabular}

$I Q R$, Interquartile range; $C O P D$, chronic obstructive pulmonary disease; $T I A$, transient ischemic attack; $M F S$, Marfan syndrome; $A I$, aortic insufficiency; $N Y H A$, New York Heart Association.

was used to calculate the adjusted hazard ratios (HRs) with $95 \%$ CI by adjusting for age, sex, coronary artery disease, preoperative severe aortic insufficiency, previous cardiac surgery, and cardiac tamponade, based on the significantly different preoperative conditions between the root repair and root replacement groups of long-term survival. A $P$ value $<.05$ (2-tailed) was considered statistically significant.

TABLE 2. Intraoperative data

\begin{tabular}{|c|c|c|c|c|c|}
\hline Variable & $\begin{array}{c}\text { Total } \\
(n=94)\end{array}$ & $\begin{array}{l}\text { Root repair } \\
(\mathrm{n}=\mathbf{4 5})\end{array}$ & $\begin{array}{l}\text { Root replacement } \\
\qquad(\mathrm{n}=39)\end{array}$ & $\begin{array}{l}\text { No root procedure } \\
\qquad(\mathbf{n}=\mathbf{1 0})\end{array}$ & $\begin{array}{c}P \\
\text { value }\end{array}$ \\
\hline Cannulation, n (\%) & & & & & .715 \\
\hline Right axillary artery & $47(50)$ & $22(49)$ & $21(54)$ & $4(40)$ & \\
\hline Innominate artery & $25(26)$ & $11(24)$ & $11(28)$ & $3(30)$ & \\
\hline Intrathoracic right subclavian artery & $11(12)$ & $7(16)$ & $3(8)$ & $1(10)$ & \\
\hline Right common carotid artery & $2(2)$ & $2(4)$ & $0(0)$ & $0(0)$ & \\
\hline Innominate and femoral arteries & $2(2)$ & $1(2)$ & $1(3)$ & $0(0)$ & \\
\hline Right axillary and femoral arteries & $1(1)$ & $1(2)$ & $0(0)$ & $0(0)$ & \\
\hline Femoral artery & $5(5)$ & $1(2)$ & $2(5)$ & $2(20)$ & \\
\hline Aorta & $1(1)$ & $0(0)$ & $1(3)$ & $0(0)$ & \\
\hline Location of primary tear, $\mathrm{n}(\%)$ & & & & & .087 \\
\hline Root & $5(5)$ & $0(0)$ & $5(13)$ & $0(0)$ & \\
\hline Ascending & $63(67)$ & $30(67)$ & $27(69)$ & $6(60)$ & \\
\hline Arch & $14(15)$ & $8(18)$ & $4(10)$ & $2(20)$ & \\
\hline Proximal descending & $7(7)$ & $5(11)$ & $2(5)$ & $0(0)$ & \\
\hline Unknown & $5(5)$ & $2(4)$ & $1(3)$ & $2(20)$ & \\
\hline Aortic valve repair, $\mathrm{n}(\%)$ & & & & & 1.000 \\
\hline Annuloplasty & $3(3)$ & $3(7)$ & $0(0)$ & $0(0)$ & \\
\hline Free margin plication & $3(3)$ & $3(7)$ & $0(0)$ & $0(0)$ & \\
\hline Arch replacement (branch vessel reimplantation), $\mathrm{n}(\%)$ & & & & & .049 \\
\hline None & $8(9)$ & $0(0)$ & $6(15)$ & $2(20)$ & \\
\hline Transverse arch ( 0 branch vessel) & $41(44)$ & $21(47)$ & $17(44)$ & $3(30)$ & \\
\hline Zone 1 arch (1-2 branch vessel) & $11(12)$ & $5(11)$ & $6(15)$ & $0(0)$ & \\
\hline Zone $2 \operatorname{arch}(2-3$ branch vessels $)$ & $26(28)$ & $15(33)$ & $7(18)$ & $4(40)$ & \\
\hline Zone $3 \operatorname{arch}(3-4$ branch vessels) & $8(9)$ & $4(9)$ & $3(8)$ & $1(10)$ & \\
\hline Frozen elephant trunk & $14(15)$ & $9(20)$ & $4(10)$ & $1(10)$ & .218 \\
\hline \multicolumn{6}{|l|}{ Concomitant procedures } \\
\hline CABG, $n(\%)$ & $4(4)$ & $0(0)$ & $4(10)$ & $0(0)$ & .043 \\
\hline TV repair, $\mathrm{n}(\%)$ & $1(10)$ & $0(0)$ & $1(3)$ & $0(0)$ & .464 \\
\hline CPB time, min, median (IQR) & $215(176-272)$ & $180(153-220)$ & $280(220-336)$ & $195(152-266)$ & $<.0001$ \\
\hline Clamp time, min, median (IQR) & $143(103-199)$ & $113(97-139)$ & $204(172-242)$ & $81(62-90)$ & $<.0001$ \\
\hline HCA time, min, median (IQR) & $25(20-30)$ & $27(22-30)$ & $21(17-30)$ & $30(22-36)$ & .023 \\
\hline Lowest temperature, ${ }^{\circ} \mathrm{C}$, median (IQR) & $24(20-28)$ & $24(20-26)$ & $24(20-28)$ & $21(20-28)$ & .472 \\
\hline Blood transfusion (PRBCs), n (\%) & & & & & .237 \\
\hline 0 units & $33(35)$ & $18(40)$ & $12(31)$ & $3(30)$ & \\
\hline 1 unit & $4(4)$ & $2(4)$ & $2(5)$ & $0(0)$ & \\
\hline 2 units & $14(15)$ & $10(22)$ & $4(10)$ & $0(0)$ & \\
\hline$\geq 3$ units & $43(46)$ & $15(33)$ & $21(54)$ & $7(70)$ & \\
\hline
\end{tabular}

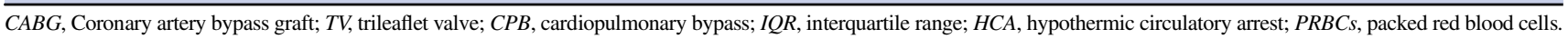


TABLE 3. Postoperative outcomes

\begin{tabular}{|c|c|c|c|c|c|}
\hline Variables & $\begin{array}{c}\text { Total } \\
(n=94)\end{array}$ & $\begin{array}{c}\text { Root repair } \\
(n=45)\end{array}$ & $\begin{array}{c}\text { Root replacement } \\
(\mathbf{n}=\mathbf{3 9})\end{array}$ & $\begin{array}{l}\text { No root procedure } \\
\quad(\mathbf{n}=\mathbf{1 0})\end{array}$ & $\begin{array}{c}P \\
\text { value }\end{array}$ \\
\hline Postoperative bleeding necessitating reoperation, $\mathrm{n}(\%)$ & $3(3)$ & $2(4)$ & $1(3)$ & $0(0)$ & 1.000 \\
\hline Myocardial infarction, $\mathrm{n}(\%)$ & $0(0)$ & $0(0)$ & $0(0)$ & $0(0)$ & \\
\hline VAD or ECMO, n (\%) & $2(2)$ & $0(0)$ & $2(5)$ & $0(0)$ & .213 \\
\hline Pacemaker, n (\%) & $0(0)$ & $0(0)$ & $0(0)$ & $0(0)$ & \\
\hline $\begin{array}{l}\text { New-onset stroke, n (\%) } \\
\text { Permanent } \\
\text { Temporary }\end{array}$ & $\begin{array}{l}4(4) \\
1(1) \\
3(3)\end{array}$ & $\begin{array}{l}3(7) \\
1(2) \\
2(4)\end{array}$ & $\begin{array}{l}1(3) \\
0(0) \\
1(3)\end{array}$ & $\begin{array}{l}0(0) \\
0(0) \\
0(0)\end{array}$ & .620 \\
\hline New-onset paraplegia, n (\%) & $1(1)$ & $0(0)$ & $1(3)$ & $0(0)$ & .464 \\
\hline $\begin{array}{l}\text { New-onset renal failure (dialysis), n (\%) } \\
\text { Permanent dialysis } \\
\text { Temporary dialysis } \\
\text { Other (died in hospital), n (\%) }\end{array}$ & $\begin{array}{l}12(13) \\
2(2) \\
6(6) \\
4(4)\end{array}$ & $\begin{array}{l}5(11) \\
1(2) \\
2(4) \\
2(4)\end{array}$ & $\begin{array}{l}4(10) \\
1(3) \\
2(5) \\
1(3)\end{array}$ & $\begin{array}{l}3(30) \\
0(0) \\
2(20) \\
1(10)\end{array}$ & 1.000 \\
\hline Respiratory failure, $\mathrm{n}(\%)$ & $11(12)$ & $6(13)$ & $4(10)$ & $1(10)$ & .745 \\
\hline Sepsis, n (\%) & $4(4)$ & $2(4)$ & $1(3)$ & $1(10)$ & 1.000 \\
\hline Gastrointestinal bleeding, $\mathrm{n}(\%)$ & $3(3)$ & $2(4)$ & $0(0)$ & $1(10)$ & .450 \\
\hline Time to extubation, h, median (IQR) & $29(12-99)$ & $24(12-56)$ & $21(11-109)$ & $82(29-156)$ & .765 \\
\hline Length of stay, d, median (IQR) & $10(7-16)$ & $10(7-15)$ & $9(6-14)$ & $16(8-28)$ & .724 \\
\hline Intraoperative mortality, $\mathrm{n}(\%)$ & $0(0)$ & $0(0)$ & $0(0)$ & $0(0)$ & \\
\hline 30-d mortality, n (\%) & $4(4)$ & $2(4)$ & $2(5)$ & $0(0)$ & 1.000 \\
\hline
\end{tabular}

$V A D$, Ventricular assist device; $E C M O$, extracorporeal membrane oxygenation; $I Q R$, interquartile range.

\section{RESULTS}

\section{Demographic and Preoperative Data}

Our patient population was a typical cohort of sick patients with ATAAD, $67 \%$ male, with a median age of 59 years (range, 22-87 years). Three percent of patients suffered cardiac arrest from massive acute myocardial infarction (MI), $11 \%$ had rupture, $16 \%$ experienced cardiac tamponade and cardiogenic shock, and $31 \%$ suffered acute end-organ malperfusion. Preoperative comorbidities were similar in the aortic root repair and replacement groups (Table 1).

\section{Intraoperative Data}

The aortic root repair was a simpler procedure than total aortic root replacement with shorter cardiopulmonary bypass and aortic cross-clamp times. There were no significant differences in cannulation strategy, aortic arch management, or transfusion of blood products between the root repair and root replacement groups (Table 2). Thirty-five percent of patients did not receive intraoperative transfusion of packed red blood cells (PRBCs), $28 \%$ never received transfusion of PRBCs, and $18 \%$ never received transfusion of any blood products throughout the course of hospitalization.

\section{Perioperative Outcomes}

No patients experienced intraoperative mortality. The 30 -day mortality was $4 \%$, and the operative mortality (including all patients who died within 30 days and/or in the hospital after surgery) was 7\% (Table 3 ). The independent risk factors for operative mortality identified on stepwise logistic analysis included cardiac tamponade (OR, 12; 95\% CI, 1.5- 90; $P=.018)$ and acute MI (OR, 12; $95 \% \mathrm{CI}, 0.83-166 ; P=.057)$ after adjusting for age, sex, type of root procedure, and type of valve. Age and type of aortic root procedure were not significantly associated with operative mortality. There were no complications or deaths related to the technique of direct repair aortic root and arch repair without adjuncts (Table 4). There were no significant differences in the rates of stroke, MI, new- onset renal failure, and operative mortality between the aortic root repair and replacement groups (Table 3).

\section{Short-Term Outcomes}

Total follow-up time was 209 patient-years, with a mean follow-up of $22 \pm 17$ months. Almost all patients $(99 \%)$ had postoperative imaging studies, and $97 \%$ had postoperative CTA of the aorta, with total of 191 CTAs and a mean of 2.2 CTAs per patient. In the aortic root repair group, $93 \%$ patients had follow-up echocardiograms. In the aortic root replacement group, $89 \%$ patients had follow-up echocardiograms. During the follow-up period, all patients were free from pseudoaneurysm at the proximal and distal anastomoses and from reoperation based on the pathology of the proximal aorta, including the aortic root, ascending aorta, and aortic arch. In the aortic root repair group, root 
TABLE 4. Causes of operative mortality

\begin{tabular}{|c|c|c|c|c|c|c|}
\hline Group & Case & Age, $y$ & Preoperative condition & Procedure & $\begin{array}{l}\text { Interval from } \\
\text { operation } \\
\text { to death, } d\end{array}$ & Cause of death \\
\hline Root repair & 1 & 58 & $\begin{array}{l}\text { Cardiac tamponade, } \\
\text { cardiogenic shock, } \\
\text { paraplegia }\end{array}$ & $\begin{array}{l}\text { Aortic root repair, AV } \\
\text { resuspension, and } \\
\text { ascending and transverse } \\
\text { arch replacement }\end{array}$ & 2 & $\begin{array}{l}\text { Hemodynamically stable; } \\
\text { family withdrew care due to } \\
\text { paraplegia }\end{array}$ \\
\hline Root repair & 2 & 64 & $\begin{array}{l}\text { Acute stroke, right upper } \\
\text { extremity malperfusion, } \\
\text { SMA malperfusion, acute } \\
\text { severe aortic insufficiency, } \\
\text { acute heart failure, acute } \\
\text { pulmonary edema, } \\
\text { cardiogenic shock }\end{array}$ & $\begin{array}{l}\text { Aortic root repair, AV } \\
\text { resuspension, zone } 2 \text { arch } \\
\text { replacement, } \\
\text { reimplantation of } \\
\text { innominate artery, LCC, } \\
\text { FET }\end{array}$ & 3 & Multiorgan system failure \\
\hline Root repair & 3 & 61 & $\begin{array}{l}\text { Cardiac tamponade, } \\
\text { cardiogenic shock, SMA } \\
\text { malperfusion, occlusion of } \\
\text { left renal artery and left } \\
\text { common iliac artery, } \\
\text { paraplegia }\end{array}$ & $\begin{array}{l}\text { Aortic root repair, AV } \\
\text { resuspension, Ascending } \\
\text { aorta and transvers arch } \\
\text { replacement with HCA }\end{array}$ & 51 & $\begin{array}{l}\text { Necrotizing pancreatitis, } \\
\text { septic shock }\end{array}$ \\
\hline Root replacement & 4 & 24 & $\begin{array}{l}\text { Cardiac arrest, coma, acute on } \\
\text { chronic heart failure, aortic } \\
\text { root aneurysm, severe AI, } \\
\text { severe pulmonary edema, } \\
\text { severe hypertension from } \\
\text { CPR }\end{array}$ & $\begin{array}{l}\text { Aortic root and ascending } \\
\text { replacement }\end{array}$ & 4 & $\begin{array}{l}\text { Preoperative hemorrhagic } \\
\text { stroke due to severe } \\
\text { hypertension from } \\
\text { epinephrine injection } \\
\text { during CPR }\end{array}$ \\
\hline Root replacement & 5 & 57 & $\begin{array}{l}\text { Acute left main coronary } \\
\text { artery occlusion, massive } \\
\text { MI, severe left and right } \\
\text { heart failure, cardiogenic } \\
\text { shock }\end{array}$ & $\begin{array}{l}\text { Aortic root replacement, } \\
\text { ascending and transverse } \\
\text { arch aorta replacement, } \\
\text { placement of ECMO }\end{array}$ & 10 & $\begin{array}{l}\text { Severe left heart failure from } \\
\text { preoperative MI. Patient } \\
\text { refused LVAD; care } \\
\text { withdrawn }\end{array}$ \\
\hline Root replacement & 6 & 60 & $\begin{array}{l}\text { Right hemisphere stroke with } \\
\text { left hemiplegia, acute and } \\
\text { chronic renal failure, } \\
\text { bacteremia }\end{array}$ & $\begin{array}{l}\text { Aortic root replacement, zone } \\
1 \text { arch replacement, RCC, } \\
\text { RScA replacement, and } \\
\text { tricuspid valve repair }\end{array}$ & 45 & $\begin{array}{l}\text { Preoperative stroke; family } \\
\text { withdrew care }\end{array}$ \\
\hline No root procedure & 7 & 81 & $\begin{array}{l}\text { Profound shock, cardiac } \\
\text { tamponade, acute renal } \\
\text { failure, respiratory failure, } \\
\text { shock liver, severe acidosis, } \\
\text { coagulopathy }\end{array}$ & $\begin{array}{l}\text { Ascending replacement, } \\
\text { aortic valve annuloplasty }\end{array}$ & 42 & $\begin{array}{l}\text { GI bleeding; family withdrew } \\
\text { care }\end{array}$ \\
\hline
\end{tabular}

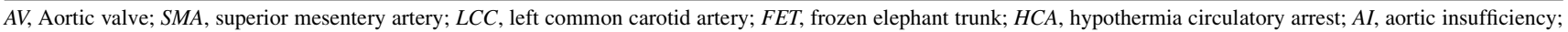
$C P R$, cardiopulmonary resuscitation; $M I$, myocardial infarction; $E C M O$, extracorporeal membrane oxygenation; $L V A D$, left ventricular assist device; $R C C$, right common carotid artery; $R S c A$, right subclavian artery; $G I$, gastrointestinal.

diameters remained between 3.8 and $4 \mathrm{~cm}$, with no significant changes noted over a 4-year period (Figure 2, $A$ ). In addition, there were no findings of residual or recurrent aortic root dissection or of severe aortic insufficiency in the aortic root repair group over the 4-year period. The severity of aortic insufficiency did not increase significantly over the 4-year period (Table 5). For the entire cohort, there was no pseudoaneurysm or significant change in the diameter of the arch (Figure 2, B); however, the proximal descending thoracic aorta started to grow in year 4 , but was not significantly enlarged (Figure 2, C). Thirty percent of patients still had a patent false lumen, $15 \%$ had a partially thrombosed false lumen, and $55 \%$ had no false lumen or a completely thrombosed false lumen. Five percent of patients underwent open repair of a descending thoracic aortic aneurysm. No patients underwent surgery for thoracoabdominal aortic aneurysm.

The 4-year Kaplan-Meier survival rate for the entire cohort was 69\% (95\% CI, 47\%-84\%) (Figure 3, A). The 4-year survival rate was significantly enhanced by the preservation of native aortic valves with aortic root repair $(\mathrm{n}=45)$ compared with root replacement with a prosthesis 
$(\mathrm{n}=24)(91 \%[95 \% \mathrm{CI}, 77 \%-96 \%]$ vs $39 \%$ [95\% CI, $11 \%-67 \%] ; P=.03$ ) (Figure 3, $B$ ). This remains true when all patients who had aortic root procedures were included by comparing patients with native valve (root repair plus the David procedure; $\mathrm{n}=60$ ) and those with a prosthesis $(\mathrm{n}=24)(92 \%$ [95\% CI, $82 \%-97 \%$ ] vs $39 \%$ $[95 \% \quad$ CI, $11 \%-67 \%] ; \quad P=.01, \quad$ log-rank test) (Figure E1). After adjusting for age, sex, coronary artery disease, preoperative severe aortic insufficiency, previous cardiac surgery, and cardiac tamponade, the risk of death was comparable (adjusted HR, 4; 95\% CI, 0.4-42; $P=.24)$ in patients undergoing aortic root replacement with a prosthesis (Bentall procedure) and those undergoing aortic root repair. There was no significant difference in 4year survival between the aortic root repair and valvesparing aortic root replacement groups $(P=.25)$.

\section{DISCUSSION}

This study examined the direct repair of the dissected aortic root and arch in patients with ATAAD without technical adjuvants including Teflon felt or biological glue. We found no complications or deaths related to the technique based on perioperative and short-term outcomes. Moreover, in all patients, the aortic root was completely healed, with no residual dissection or recurrent dissection and no pseudoaneurysm of the anastomoses for up to 5 years after repair. The aortic valve remained competent after aortic root/valve repair. Unexpectedly, we found superior 4-year survival in the aortic root repair group compared with the aortic root replacement group with a prosthesis by Kaplan-Meier analysis (Figure 3, B).

Direct repair of the dissected aortic root and arch is safe in selected patients with ATAAD. The pioneers of cardiac surgery have attempted direct repairs of dissected in ATAAD before biological glue was introduced to cardiac surgery. The operative mortality was $28 \%$ to $30 \%$ in ATAAD repair owing to numerous factors at that time, including perfusion technique, myocardial protection, preoperative and postoperative care, and other factors. ${ }^{13-15}$ The dissected aorta is extremely fragile, and the outer layer of the dissected aorta demonstrates pathological thinning. When repairing an ATAAD, surgeons fear disintegration of the anastomoses and the potential for uncontrollable bleeding. Technical adjuncts, including Teflon felt and biological glue, have been applied extensively to facilitate this life-saving procedure, but this approach also has been associated with pseudoaneurysm at the anastomoses, redissection, and aortic root aneurysm. ${ }^{2-6}$ Since the 1980s, using biological glue and Teflon felt for ATAAD repair has become dogma despite the toxicity and side effects. Based on the aforementioned risks, we have directly repaired the dissected aorta with fine suture (5-0 Prolene) without Teflon felt or biological glue or any adjuncts over the previous 5 years in 94 patients. We modified the direct repair technique using fine suture (5-0 Prolene with a small needle) and adding the reinforcement around the coronary ostia if the aortic dissection extends around the coronary ostia (Figure 1). At present, the intraoperative mortality is $0 \%$, providing an initial indication that the repairs are intact and there is no evidence of disintegration of the aorta or fine suture rupture. Thirty-five percent of our patients did not require intraoperative transfusion of PRBCs, which also supports excellent hemostasis. We report no operative deaths related to technical complications when directly repairing the aortic root and arch without Teflon felt and biological glue (Table 4). Beyond operative mortality, there have been no additional deaths reported in the aortic root repair group over the 5-year follow-up.

This approach is effective. By approximating the dissected 2 layers of the aortic root together at the STJ, we completely obliterate the false lumen of the aortic root. The coronary ostial reinforcing suture line prevents any further dissection from extending into the coronary arteries. For all patients, both the intraoperative transesophageal echocardiography immediately after repair and follow-up CTA at the 3-month visit consistently confirmed that the false lumen had been obliterated at the site of the repaired aortic root. The blood pressure inside the aortic root pushes the dissection flap against the outer layer, which keeps the dissection flap attached to the outer layer of the dissected aortic root. The small needle with the 5-0 Prolene suture also limited bleeding from the needle hole into the false lumen of the aortic root. Because there was no Teflon felt, biological glue, or thrombus in the false lumen and no intimal tear, the aortic root demonstrated long-term healing. This approach allows the native aortic root to maintain its natural geometry, which supports the competency of the aortic valve. We contend that no reconstructed aortic root with artificial materials is as good as a normal healed native aortic root in terms of the aspects of valve function and the risk of infection. After the aortic root/valve repair, the preoperative aortic insufficiency was mostly corrected to none or trivial. The competency of the aortic valve has persisted for up to 5 years (Table 5). At the distal anastomosis of the aortic arch, the repair was performed the same way with 5-0 Prolene sutures. None of our patients developed malperfusion of end organs following repair. During the 5-year follow-up, there have been no arch aneurysms or anastomotic pseudoaneurysms in the entire cohort, indicating that the repair is safe and effective. In our study, $30 \%$ of patients had a patent false lumen at the proximal descending aorta, and $5 \%$ underwent surgery for a descending thoracic aortic aneurysm during follow-up. This is consistent with the results reported by Oda and colleagues ${ }^{16}$ using inverted adventitia and Teflon felt for the distal anastomosis of the hemiarch in ATAAD as a new technique. 

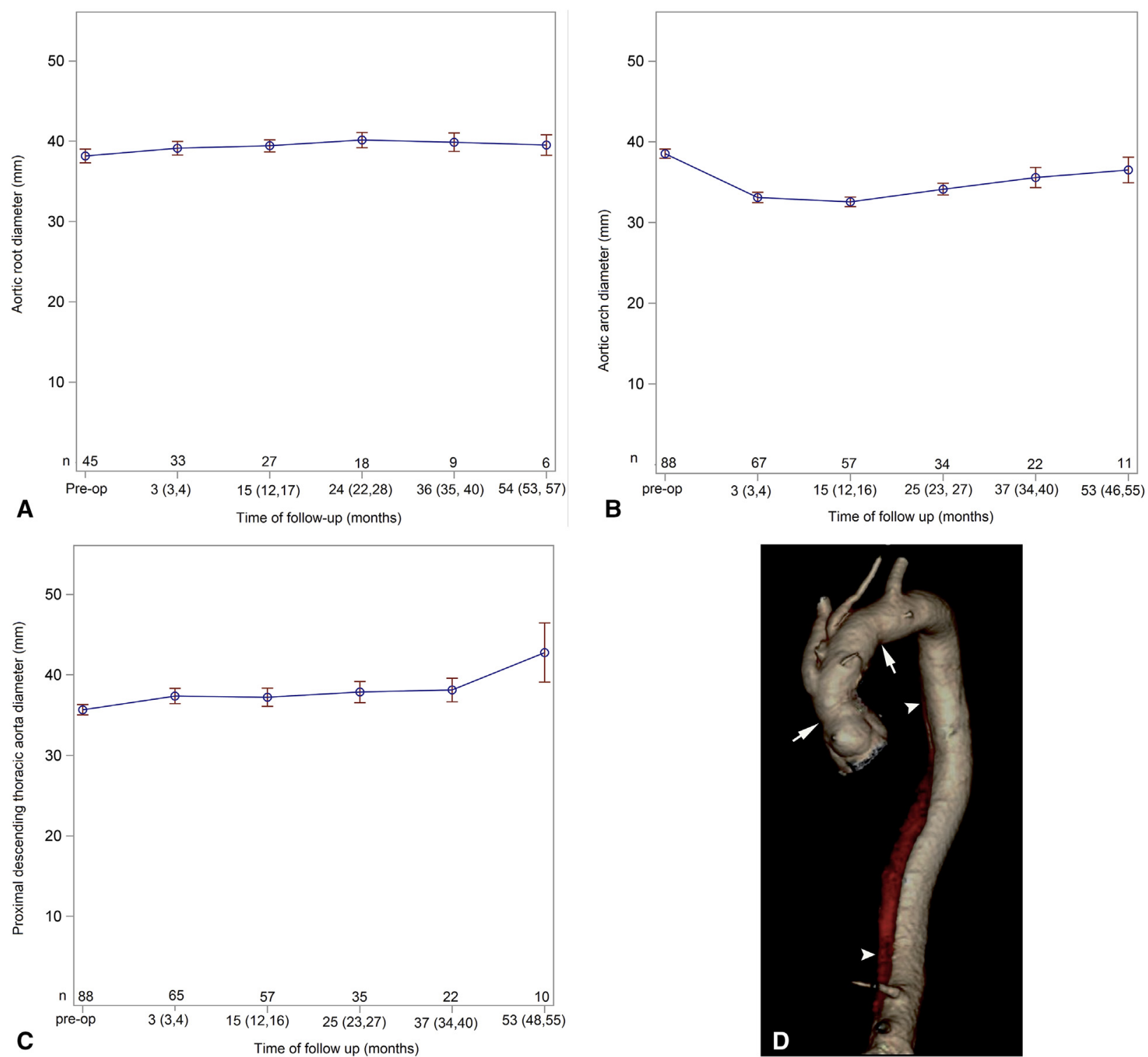

FIGURE 2. The diameters of the preserved aortic root (A), arch (B), and proximal descending thoracic aorta (C) did not change significantly during 4-year follow-up. $n$, the number of measurements of the aortic root by computed tomography angiography (CTA) or echocardiography during that time frame. (D) Follow-up CTA 3 years after the aortic root repair, zone 2 arch replacement, and replacement of innominate artery and left common carotid artery. Two arrows indicate the proximal and distal anastomoses of a multibranch Dacron graft to the dissected aorta. Between the 2 arrows, the innominate artery, left common carotid artery, and 2 stumps of branch grafts are visualized. Two arrowheads indicate the thrombosed false lumen at the proximal descending thoracic aorta.

Another group has attempted to repair the STJ by inverting the dissected outer layer (adventitia) into the true lumen of the aorta at the STJ and the arch for 18 patients, with a 30 -day mortality of $11 \% .{ }^{17}$ Because the adventitia is highly thrombogenic, we prefer to not use the adventitia as a buttress for the anastomosis, to avoid the potential risk of thromboembolism. We telescoped the Dacron graft to the aortic true lumen and directly attached the graft material to the intima of the aorta at the proximal and distal anastomotic sties, to prevent blood flow from pressurizing the surface of section of the dissected aorta at the anastomoses.

Preservation of the dissected aortic root always raises the question of recurrence of aortic root pathology, such as recurrent aortic root dissection, aneurysm/ pseudoaneurysm, severe aortic insufficiency, reoperation, and/or death. ${ }^{18}$ However, aortic valve resuspension with preservation of the aortic root in selected patients with ATAAD has been performed for 30 years, with excellent long-term results. ${ }^{8,9,19-21}$ Some of the known 
TABLE 5. Aortic valve function by echocardiogram during follow-up

\begin{tabular}{|c|c|c|c|c|c|c|c|c|c|}
\hline \multirow[b]{2}{*}{$\begin{array}{c}\text { Aortic } \\
\text { insufficiency }\end{array}$} & \multicolumn{3}{|c|}{ Root repair } & \multicolumn{3}{|c|}{ Root replacement: David procedure } & \multicolumn{3}{|c|}{ Root replacement: Bentall procedure } \\
\hline & $\begin{array}{l}\text { Preoperative } \\
\quad(n=45)\end{array}$ & $\begin{array}{c}\text { Postoperative } \\
(n=45)\end{array}$ & $\begin{array}{c}\text { Last } \\
\text { follow- up } \\
(\mathrm{n}=\mathbf{3 7})\end{array}$ & $\begin{array}{l}\text { Preoperative } \\
\quad(\mathbf{n}=15)\end{array}$ & $\begin{array}{c}\text { Postoperative } \\
\quad(\mathbf{n}=15)\end{array}$ & $\begin{array}{c}\text { Last } \\
\text { follow-up } \\
(\mathrm{n}=15)\end{array}$ & $\begin{array}{l}\text { Preoperative } \\
\quad(\mathbf{n}=\mathbf{2 4})\end{array}$ & $\begin{array}{c}\text { Postoperative } \\
\quad(n=24)\end{array}$ & $\begin{array}{c}\text { Last } \\
\text { follow-up } \\
(\mathrm{n}=18)\end{array}$ \\
\hline None & $8(18)$ & $30(67)$ & $26(70)$ & $4(27)$ & $10(67)$ & $9(60)$ & $8(33)$ & $24(100)$ & $18(100)$ \\
\hline Trace & $8(18)$ & $8(18)$ & $2(5)$ & $0(0)$ & $5(33)$ & $1(7)$ & $1(4)$ & $0(0)$ & $0(0)$ \\
\hline Mild & $19(42)$ & $7(15)$ & $9(25)$ & $4(27)$ & $0(0)$ & $5(33)$ & $3(13)$ & $0(0)$ & $0(0)$ \\
\hline Moderate & $7(16)$ & $0(0)$ & $0(0)$ & $3(20)$ & $0(0)$ & $0(0)$ & $6(25)$ & $0(0)$ & $0(0)$ \\
\hline Severe & $3(7)$ & $0(0)$ & $0(0)$ & $4(27)$ & $0(0)$ & $0(0)$ & $6(25)$ & $0(0)$ & $0(0)$ \\
\hline
\end{tabular}

complications from a preserved dissected aortic root may actually be related to the use of biological glue, ${ }^{19}$ which our approach avoids. Total root replacement can be achieved for patients with an ATAAD with low mortality in experienced hands, ${ }^{20,22}$ as shown in the present study. Nonetheless, aortic root replacement remains a challenge for many US surgeons especially in emergency situations such as ATAAD. The median number of aortic root procedures per center per year is 2 , and only $5 \%$ of centers perform $>16$ aortic root procedures annually. ${ }^{23}$ Simplifying the root procedure for select patients with ATAAD (using aortic root repair without glue or Teflon felt instead of a total root replacement) may improve survival in those patients. Our short-term results show no patients with recurrent proximal aortic pathology leading to reoperation or death in our aortic root repair group. Kaplan-Meier analysis showed significantly enhanced midterm survival, with preservation of the native aortic valve through aortic root repair (Figure 3, $B$, and Figure E1). The difference could be attributed to the preservation of native aortic valve in the root repair group and underlying aortic valve pathology in the root replacement group before the operation, given that the patients with prosthetic root replacement had chronic aortic stenosis or insufficiency due to valvulopathy or root aneurysm. However, because valvulopathy did not cause a significant change in the left ventricular ejection fraction in the root replacement group compared with the root repair group (Table 1), preservation of native valve alone may contribute to the survival benefit. In summary, our findings support that preservation of the aortic root is an acceptable approach in selected patients with ATAAD.

This study has some important limitations, including small sample size, limited follow-up period, observational study design, lack of randomization of treatments, and experience based on a single surgeon and single center. We acknowledge that these factors can introduce bias in patient and treatment selection. Furthermore, the absence of statistical significance might not properly reflect clinical significance due to the small cohort size and rarity of postoperative events. This is particularly relevant when subgroups within the overall cohort are compared. In addition, long-term follow-up is needed. However, the formation of pseudoaneurysms at the anastomosis typically occurs within 1 to 65 months (mean, 30 months) following ATAAD, as reported in cases using biological glue. $^{5}$ We did not observe this complication for up to 65 months.
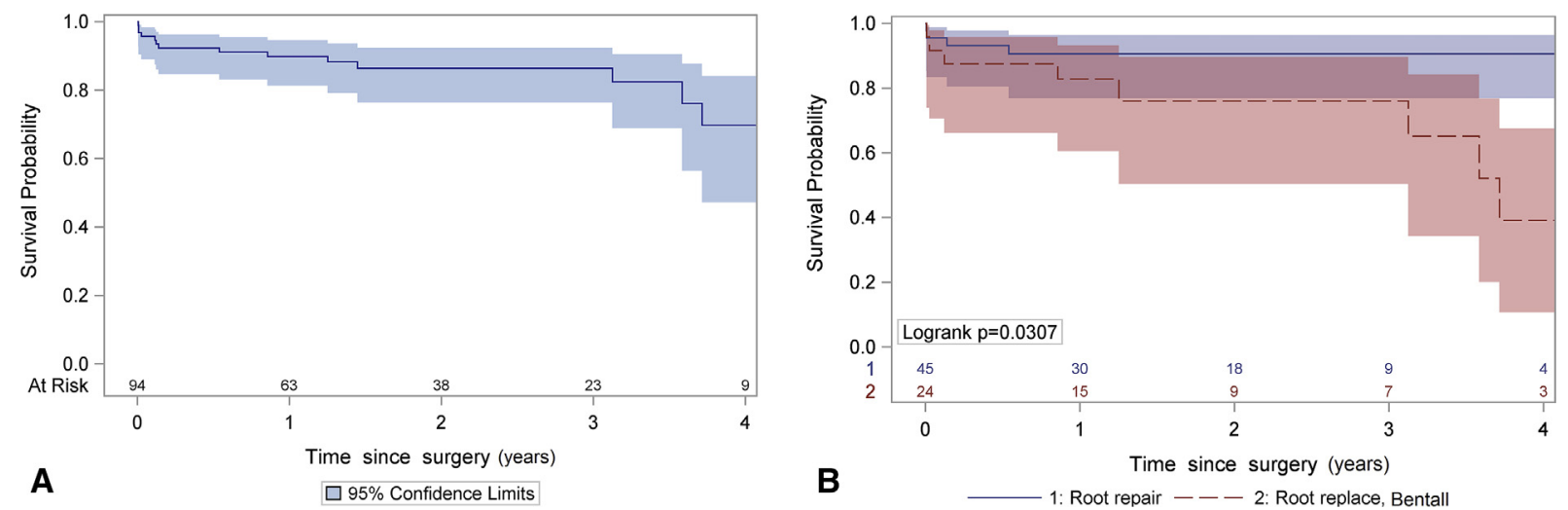

FIGURE 3. Kaplan-Meier survival curves for the whole cohort at 4 years (A) and for the root repair group versus aortic root replacement with prosthesis (Bentall) at 4 years (B). 


\section{CONCLUSIONS}

Direct repair of the aortic root and arch by fine suture approximation of the aortic wall to close the false lumen without use of technical adjuncts, such as Teflon felt or biological glue, or resection of dissected aortic sinuses is safe and effective, with favorable short-term results. We recommend direct aortic root repair for patients with ATAAD suitable for aortic root preservation, and direct arch anastomosis without any technical adjuncts for all patients with ATAAD. If possible, preservation of the native aortic valve should be considered for a potential survival benefit.

\section{Conflict of Interest Statement}

Authors have nothing to disclose with regard to commercial support.

We thank Drs Himanshu Patel, Michael Deeb, and Richard Prager, Department of Cardiac Surgery, University of Michigan, for their support.

\section{References}

1. Berretta P, Patel HJ, Gleason TG, Sundt TM, Myrmel T, Desai N, et al. IRAD experience on surgical type A acute dissection patients: results and predictors of mortality. Ann Cardiothorac Surg. 2016;5:346-51.

2. Hata H, Takano H, Matsumiya G, Fukushima N, Kawaguchi N, Sawa Y. Late complications of gelatin-resorcin-formalin glue in the repair of acute type A aortic dissection. Ann Thorac Surg. 2007;83:1621-6.

3. Suzuki S, Masuda M, Imoto K. The use of surgical glue in acute type A aortic dissection. Gen Thorac Cardiovasc Surg. 2014;62:207-13.

4. Izutani H, Shibukawa T, Kawamoto J, Ishibashi K, Nishikawa D. Devastating late complication for repair of type A acute aortic dissection with usage of gelatin-resorcinol-formalin glue. Interact Cardiovasc Thorac Surg. 2007;6:240-2.

5. Suzuki S, Imoto K, Uchida K, Takanashi Y. Aortic root necrosis after surgical treatment using gelatin-resorcinol-formaldehyde (GRF) glue in patients with acute type A aortic dissection. Ann Thorac Cardiovasc Surg. 2006;12:333-40.

6. Bingley JA, Gardner MA, Stafford EG, Mau TK, Pohlner PG, Tam RK, et al. Late complications of tissue glues in aortic surgery. Ann Thorac Surg. 2000;69: 1764-8.

7. Karck M. The glue crisis in surgery of acute aortic dissection type A is ongoing. Eur J Cardiothorac Surg. 2012;41:e36-7.

8. Rylski B, Bavaria JE, Milewski RK, Vallabhajosyula P, Moser W, Kremens E, et al. Long-term results of neomedia sinus valsalva repair in 489 patients with type A aortic dissection. Ann Thorac Surg. 2014;98:582-8; discussion 588-9.

9. Miller DC. Surgical treatment of aortic dissections. In: Cardiac Surgery. Rob and Smith's Operative Surgery. In: Jamieson SW, Shumway NE, eds7. London: Butterworth; 1986:526-37.
10. Centers for Disease Control and Prevention; National Center for Health Statistics. National Death Index. Available at: https://www.cdc.gov/nchs/ndi/ index.htm. Accessed December 27, 2017.

11. Kunihara T, Neumann N, Kriechbaum SD, Aicher D, Schäfers HJ. Aortic root remodeling leads to good valve stability in acute aortic dissection and preexistent root dilatation. J Thorac Cardiovasc Surg. 2016;152:430-6.e1.

12. Leshnower BG, Myung RJ, McPherson L, Chen EP. Midterm results of David V valve-sparing aortic root replacement in acute type A aortic dissection. Ann Thorac Surg. 2015;99:795-800; discussion 800-1.

13. Daily PO, Trueblood HW, Stinson EB, Wuerflein RD, Shumway NE Management of acute aortic dissections. Ann Thorac Surg. 1970;10 237-47.

14. Miller DC, Stinson EB, Oyer PE, Rossiter SJ, Reitz BA, Griepp RB, et al. Operative treatment of aortic dissections. Experience with 125 patients over a sixteen-year period. J Thorac Cardiovasc Surg. 1979;78:365-82.

15. Debakey ME, Henly WS, Cooley DA, Morris GC Jr, Crawford ES, Beall AC Jr. Surgical management of dissecting aneurysms of the aorta. J Thorac Cardiovasc Surg. 1965;49:130-49.

16. Oda T, Minatoya K, Sasaki H, Tanaka H, Seike Y, Itonaga T, et al. Adventitial inversion technique for type A aortic dissection distal anastomosis. J Thorac Cardiovasc Surg. 2016:151:1340-5.

17. Tanaka K, Morioka K, Li W, Yamada N, Takamori A, Handa M, et al. Adventitial inversion technique without the aid of biologic glue or Teflon buttress for acute type A aortic dissection. Eur J Cardiothorac Surg. 2005;28:864-9.

18. Saczkowski R, Malas T, Mesana T, de Kerchove L, El Khoury G, Boodhwani M. Aortic valve preservation and repair in acute Type A aortic dissection. Eur J Cardiothorac Surg. 2014:45:e220-6.

19. von Oppell UO, Karani Z, Brooks A, Brink J. Dissected aortic sinuses repaired with gelatin-resorcin-formaldehyde (GRF) glue are not stable on follow up. $J$ Heart Valve Dis. 2002;11:249-57.

20. Urbanski PP, Lenos A, Irimie V, Bougioukakis P, Zacher M, Diegeler A Acute aortic dissection involving the root: operative and long-term outcome after curative proximal repair. Interact Cardiovasc Thorac Surg. 2016;22:620-6.

21. Fann JI, Glower DD, Miller DC, Yun KL, Rankin JS, White WD, et al. Preservation of aortic valve in type A aortic dissection complicated by aortic regurgitation. J Thorac Cardiovasc Surg. 1991;102:62-73; discussion 73-5.

22. Graeter TP, Langer F, Nikoloudakis N, Aicher D, Schäfers HJ. Valve-preserving operation in acute aortic dissection type A. Ann Thorac Surg. 2000;70: $1460-5$.

23. Stamou SC, Williams ML, Gunn TM, Hagberg RC, Lobdell KW, Kouchoukos NT. Aortic root surgery in the United States: a report from the Society of Thoracic Surgeons database. J Thorac Cardiovasc Surg. 2015;149: 116-22.e4.

Key Words: acute type A aortic dissection, aortic root repair, aortic root replacement, aortic arch replacement, operative outcome, biological glue 


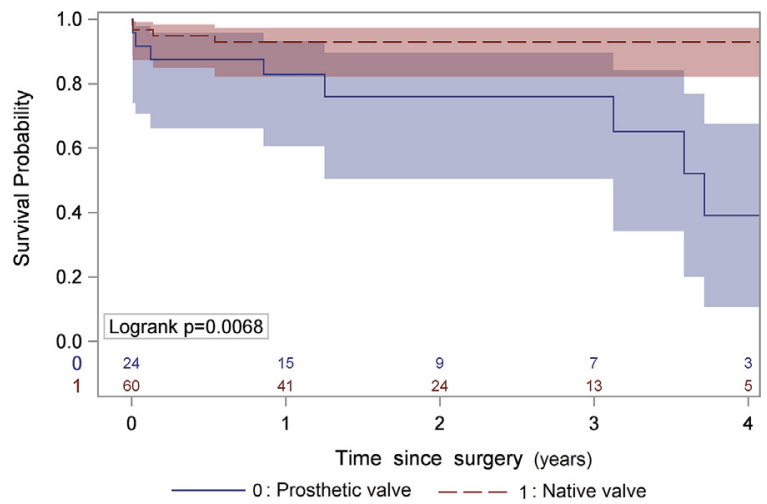

FIGURE E1. Kaplan-Meier survival curve comparing patients with native valve (aortic root repair plus the David procedure; $\mathrm{n}=60$ ) and those who underwent the Bentall procedure $(\mathrm{n}=24)$.
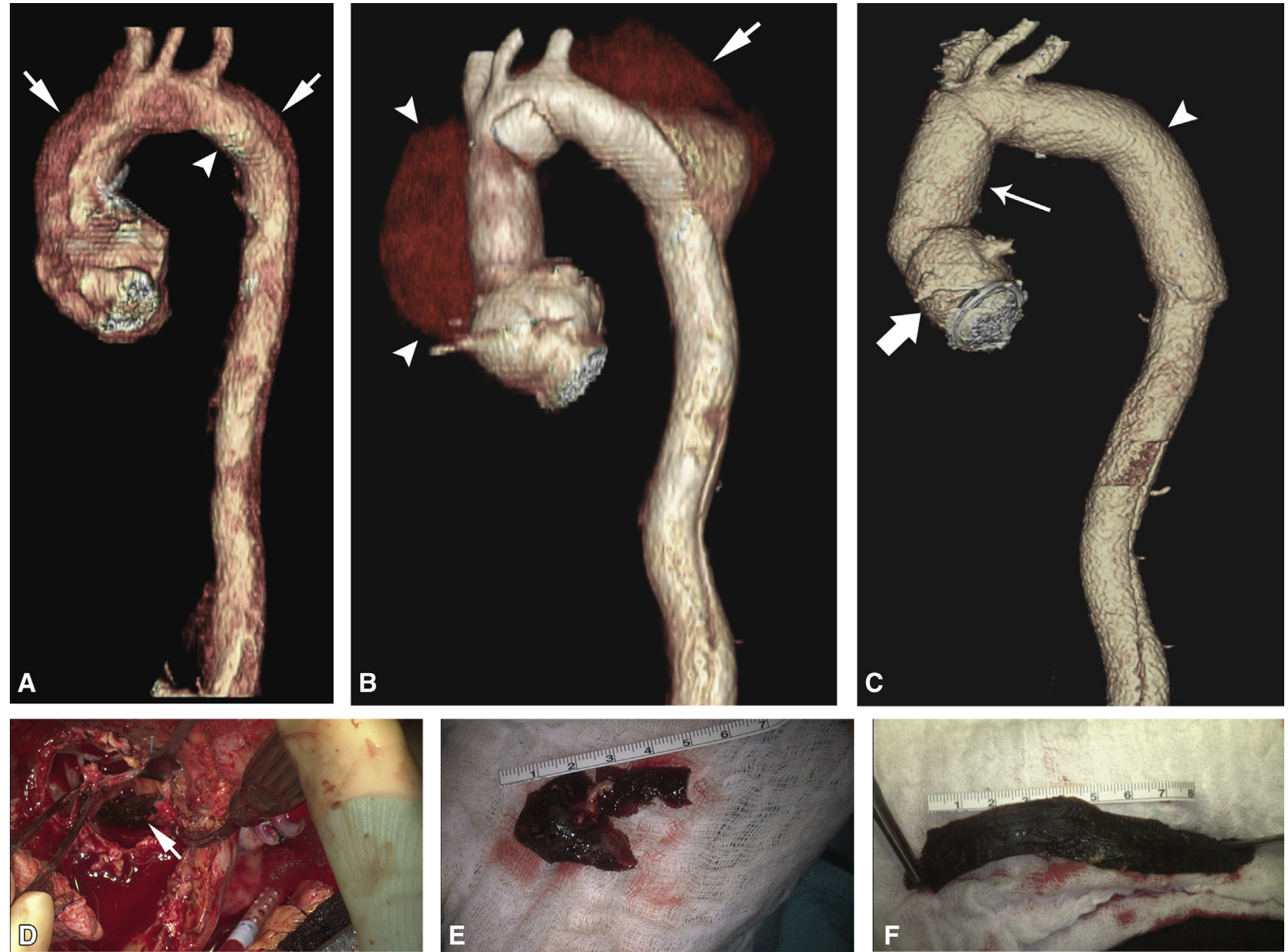

FIGURE E2. In April 2016, a 50-year-old male who had acute type A aortic dissection (ATAAD) repair at another hospital with BioGlue and Teflon felt. A, A 3D volume rendering (VR) image from CTA of ATAAD with true (arrowheads) and false (arrows) lumens. B, A 3D VR image at 2.5 months after repair of ATAAD with BioGlue and Teflon felt. Note that pseudoaneurysms in the ascending (between arrowheads) and proximal descending (arrow) thoracic aorta where the BioGlue was injected into the false lumen. C, We performed 2-stage repair of aortic root aneurysm (Bentall procedure) and ascending and descending aortic pseudoaneurysms. The mechanical composite valve graft for the aortic root replacement (thick arrow) and the Dacron graft for the ascending/transverse arch (thin arrow) and reverse hemiarch/descending aortic pseudoaneurysm replacement (arrowhead) are noted. D, BioGlue injected into the false lumen of aortic root (arrow) at first ATAAD repair at outside hospital. E, BioGlue removed from the false lumen of aortic root. F, BioGlue removed from the false lumen of the distal aortic arch and proximal descending aorta. 